\title{
Exergy-based analysis of irreversibilities for heat pump working fluids and cycle layouts
}

Zühlsdorf, Benjamin; Jensen, Jonas Kjær; Elmegaard, Brian

Published in:

Proceedings of ECOS 2019: 32nd International Conference on Efficiency, Cost, Optimization, Simulation and Environmental Impact of Energy Systems

Publication date:

2019

Document Version

Publisher's PDF, also known as Version of record

Link back to DTU Orbit

Citation (APA):

Zühlsdorf, B., Jensen, J. K., \& Elmegaard, B. (2019). Exergy-based analysis of irreversibilities for heat pump working fluids and cycle layouts. In Proceedings of ECOS 2019: 32nd International Conference on Efficiency, Cost, Optimization, Simulation and Environmental Impact of Energy Systems

\section{General rights}

Copyright and moral rights for the publications made accessible in the public portal are retained by the authors and/or other copyright owners and it is a condition of accessing publications that users recognise and abide by the legal requirements associated with these rights.

- Users may download and print one copy of any publication from the public portal for the purpose of private study or research.

- You may not further distribute the material or use it for any profit-making activity or commercial gain

- You may freely distribute the URL identifying the publication in the public portal 


\title{
Exergy-based analysis of irreversibilities for heat pump working fluids and cycle layouts
}

\author{
Benjamin Zühlsdorfa,b, Jonas Kjar Jensen $^{a}$, Brian Elmegaard ${ }^{a}$ \\ ${ }^{a}$ Department of Mechanical Engineering, Technical University of Denmark, Kgs. Lyngby, Denmark, \\ jkjje@mek.dtu.dk, be@mek.dtu.dk \\ ${ }^{b}$ Energy and Climate, Danish Technological Institute, Aarhus, Denmark, bez@dti.dk
}

\begin{abstract}
:
The use of zeotropic mixtures in heat pumps in well-designed cycles enables considerable performance improvements beyond the limitations of conventional systems. A beneficial working fluid choice enables matching the temperature profiles of the working fluid and the secondary media. The reduced temperature differences in the heat exchangers decrease the exergy destruction and yield higher cycle performance. Further improvements may be obtained from installing internal heat exchangers and reducing the superheating inside the evaporator.

Conducting an advanced exergy analysis for the evaluation of different cycle layouts and working fluids yields varying amounts of unavoidable exergy destruction depending on the scenario. These differences in unavoidable exergy destruction may be considered as the performance improvement potential obtainable by an optimization of the working fluid and the cycle layout. This study therefore suggests splitting the exergy destruction into two contributions, of which one is associated with the heat exchanger operating with an ideal fluid and the other with the fluid being non-ideal. Furthermore, the exergy destruction was related to deviations from the Lorenz cycle in terms of COP. The suggested concept was demonstrated by the case of a heat pump with different mixtures of propane/butane at different cycle layouts. The results were found to be a suitable measure of the potential performance improvement obtainable by working fluid and cycle layout optimization. Furthermore, relating the exergy destruction to changes in COP enabled an intuitive interpretation of the results.
\end{abstract}

\section{Keywords:}

Cycle design, Temperature glide matching, Zeotropic working fluid mixtures.

\section{Introduction}

Heat pumps enable sustainable and electricity-based heat supply in several applications, such as domestic heating or supply of heat to industrial process or district heating networks. The thermodynamic performance of heat pumps is strongly dependent on the working fluid and the cycle layout. The choice of the working fluid is furthermore subject to legislative restrictions ensuring a limited environmental impact of the working fluid, which facilitates the search for suitable alternatives. 
The choice of the working fluid and the cycle layout accordingly became the focus of various recent studies. Domanski et al. [1] studied the limitations for hypothetical pure fluids and outlined the fundamental trade-off among capacity and thermodynamic performance. McLinden et al. [2] complemented this study by an analysis of potential real pure fluids. Recently, McLinden et al. [3] concluded the range of promising replacement fluids to be rather limited. They furthermore outlined the dependency of the cycle layout and the working fluid with respect to an optimal thermodynamic performance.

Harby [4] reviewed the possibilities for using mixtures of hydrocarbons as replacement fluids and indicated an increased flexibility considering a limited number of pure fluids, which are suitable as long-term replacements. Zühlsdorf et al. [5] analysed different approaches for comparing working fluids and suggested a procedure for identifying high-performance working fluids. It was demonstrated that zeotropic working fluid mixtures could yield a considerable increase in thermodynamic performance in applications in which the heat source and heat sink are experiencing a temperature glide. The improvements could mainly be associated with an improved match of the temperature profiles of the heat source and sink with the working fluid. It was furthermore found that the potential performance increase was dominated by the glide match in the source [6] and that the optimal cycle design and the optimal working fluid were interdependent. The presented approaches did however require a sophisticated procedure for the selection of the working fluid.

Other studies focused on the development of a more advanced cycle layout, which enabled adjustment of the temperature glide of just one mixture and thereby obtaining a similar effect. Jensen et al. [7] studied the performances of hybrid ammonia-water absorption-compression heat pumps and outlined different advantages, such as higher heat supply temperatures and improved thermodynamic performances. Van de Bor et al. [8] studied the integration of a compressionresorption heat pump into various case studies and confirmed optimal thermodynamic performances for good temperature glide matches. Gudjonsdottir et al. [9] studied the impact of adding carbon dioxide to a mixture of ammonia and water in a wet compression-resorption heat pump cycle and found different advantageous aspects, such as a decreased pressure ratio and increased overall performances.

These studies document that the combined optimization of the cycle layout and the working fluid may lead to considerable performance increases. Exploiting these potentials does however require sophisticated methods for the evaluation of different alternatives.

Advanced exergy analysis [10-12] is a suitable tool for quantifying the interdependencies among the components, e.g., to describe the impact of the compressor efficiency on the condenser performance, by distinguishing endogenous and exogenous contributions. It is furthermore suitable for the quantification of the maximum performances for a given system using components of different efficiencies, e.g., to determine what efficiency could be reached with an infinite heat exchanger area, by distinguishing between an avoidable and unavoidable contribution [13]. Kelly et al. [14] compared different approaches for the calculation of the endogenous and exogenous contributions to the exergy destruction, enabling association of the exergy destruction with the component where it was caused rather than with the component in which it occurred.

Morosuk and Tsatsaronis [15] conducted an advanced exergy analysis for an absorption refrigeration machine. Suggestions for the optimization of the system were derived from the results and found to be more comprehensive than comparable studies. Jensen et al. [16] performed an exergoeconomic optimization of a hybrid ammonia-water absorption-compression heat pump for heat supply in a spray drying facility for finding the design with an optimal economic performance.

From the review of the advanced exergy analysis in refrigeration and heat pump systems it may be concluded that advanced exergy analysis is generally a suitable method for evaluating the performance and the limitations of a given system.

Morosuk and Tsatsaronis [17] applied advanced exergy analysis for evaluating a set of working fluids for a refrigeration cycle. It was observed that the share of unavoidable exergy destruction in refrigeration machines depended on the working fluid. In the context of optimizing the cycle 
performance by the working fluid choice, these variations may be understood as the potential improvements obtainable by the choice of an optimal working fluid. Morosuk and Tsatsaronis [17] indicated moderate improvement potentials for the analysed case, while it was shown in e.g., $[5,6,18]$ that these improvement potentials may become considerable in applications with large temperature glides and for the utilization of zeotropic working fluid mixtures with a good temperature glide match.

This paper therefore presents an analysis of the impact of the working fluid choice on the exergy destruction. Zühlsdorf et al. [6] suggested distinguishing between a contribution that is associated with the heat exchanger and one contribution that is associated with the choice of the working fluid and the cycle layout. Furthermore, a relation is derived to describe the dependency between the exergy destruction and the coefficient of performance COP, enabling an intuitive interpretation of the saving potentials and quantifying the deviations from an ideal theoretical cycle by different contributions of exergy destruction.

\section{Methods}

The presented analysis was conducted for a heat pump system for which pure working fluids and zeotropic working fluid mixtures were evaluated for two different cycle layouts. In the following, the considered cycle layouts and the corresponding modelling assumptions are explained. Subsequently, an approach for splitting the exergy analysis into different contributions is presented, before the relation between the exergy destruction and the COP is derived.

\subsection{Heat pump cycles and working fluids}

Two different cycle layouts are considered during the analysis. Figure 1 shows a standard (std) cycle in which the working fluid receives heat from the heat source at a low pressure, before it is compressed to reject heat to the heat sink. In the standard cycle, the working fluid is subsequently throttled to the low pressure. Figure 2 shows the layout of a cycle with an internal heat exchanger (IHX), in which the working fluid is further subcooled before the throttling valve, while preheating the suction line of the compressor. The internal heat exchanger allows furthermore reducing the superheating inside the evaporator and possibly shifting some of the evaporation process to the internal heat exchanger. The optimal definition of the cycle depends on the case and the selected medium and will be analyzed for specific examples.

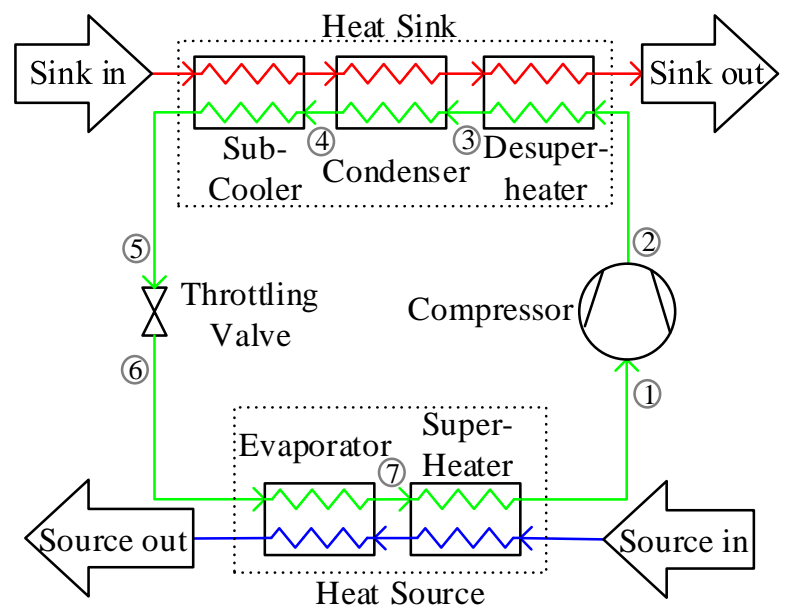

Figure 1. Standard heat pump cycle (std)

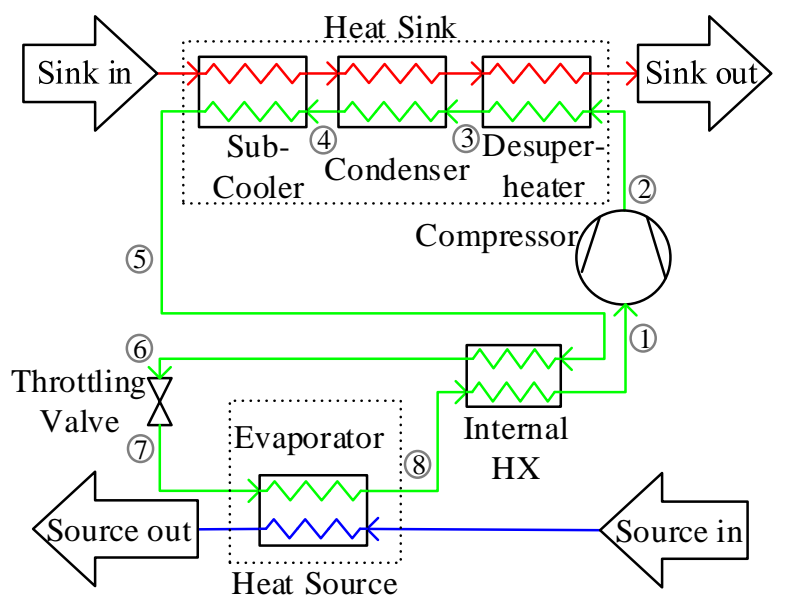

Figure 2. Heat pump cycle with internal heat exchanger (IHX)

The pressure levels were defined by minimum pinch point temperature differences in the heat exchangers of $2.5 \mathrm{~K}$. The outlet temperature of the subcooler was defined by the pinch point temperature difference to the sink inlet temperature, as this yielded a maximum subcooling and the highest thermodynamic performance. The compressor was modelled with an isentropic efficiency of 
$80 \%$ and no heat losses to the environment were considered. No losses were considered for the motor.

As the main indicator of the thermodynamic performance, the coefficient of performance COP was introduced as the ratio of the supplied heat $\dot{Q}_{\text {Sink }}$ and the power supplied to the compressor $\dot{W}_{\text {Comp }}$.

$$
\mathrm{COP}=\frac{\dot{Q}_{\text {Sink }}}{\dot{W}_{\text {Comp }}}
$$

As an indicator of the size of the compression equipment and thereby of the investment cost, the volumetric heating capacity VHC was considered. It relates the supplied heat flow rate $\dot{Q}_{\text {Sink }}$ to the volume flow rate at the suction line of the compressor $\dot{V}_{1}$.

$$
\mathrm{VHC}=\frac{\dot{Q}_{\text {Sink }}}{\dot{V}_{1}}
$$

The numerical models were based on mass and energy balances and accessed medium properties from Refprop [19]. The numerical models are documented and accessible at [20].

In this study, the analysis focussed on the optimization of the working fluid choice in combination with the cycle layout. Therefore, propane (R-290), butane (R-600) and all possible binary mixtures of these were considered. Furthermore, the impact of the internal heat exchanger and the state of the working fluid at the outlet of the evaporator were analysed.

The analysis is presented for a case study in which a heat sink of constant capacity was heated from $40{ }^{\circ} \mathrm{C}$ to $70{ }^{\circ} \mathrm{C}$, while a heat source of constant capacity was cooled from $20{ }^{\circ} \mathrm{C}$ to $10{ }^{\circ} \mathrm{C}$. The supplied heat rate was maintained throughout the analysis.

\subsection{Associating exergy destruction due to heat transfer with component or cycle layout and working fluid}

Recent studies on the optimization of heat pump systems by selecting the working fluid and the cycle layout have demonstrated a considerable potential for improvements in thermodynamic performance $[5,6,18]$. This optimization approach focused to some extent on the reduction of what was considered as unavoidable exergy destruction by advanced exergy analysis [17]. While advanced exergy analysis was found to be suitable for identifying the amount of unavoidable exergy destruction for a pre-defined system, it did not include further conclusions about what amount of exergy destruction could be omitted by optimising the cycle layout and the working fluid.

This section introduces a concept to associate part of the exergy destruction with the component and the remaining part with the working fluid and the cycle layout [6,21]. This approach was derived and demonstrated by the example of vapour compression heat pumps using pure and mixed refrigerants. Vapour compression heat pumps and refrigeration systems typically comprise a heat exchange process in which heat is obtained at a low temperature from the heat source, and one in which heat is rejected at higher temperature to the heat sink. These heat exchange processes do however cause a certain amount of exergy destruction which could not be avoided even with an ideal fluid and another part accounting for the fluid deviating from being ideal.

Figure 3 shows the temperature-heat-diagram of the standard cycle using butane for an exemplifying application. The temperature profiles of the working fluid during evaporation and condensation do not match with the temperature profiles of source and sink. The diagram includes furthermore the areas highlighting the temperature mismatches, which are causing the exergy destruction due to heat transfer. Aiming to reduce the temperature differences within the heat exchanger, and thereby the exergy destruction, while respecting a minimum pinch point temperature difference yields a balanced heat exchanger as the optimal process and is depicted by the ideal fluid in the temperature-heat-diagram. The balanced heat exchanger defines the amount of exergy 
destruction $\dot{E}_{\mathrm{D} \text {,pinch }}$, which is associated with the heat exchanger equipment and considered to be inevitable even with an ideal fluid. The temperature profile of butane is deviating from the ideal process, which yields an increased mismatch and accordingly the exergy destruction that can be associated with the fluid being non-ideal $\dot{E}_{\mathrm{D}, \text { fluid }}$.

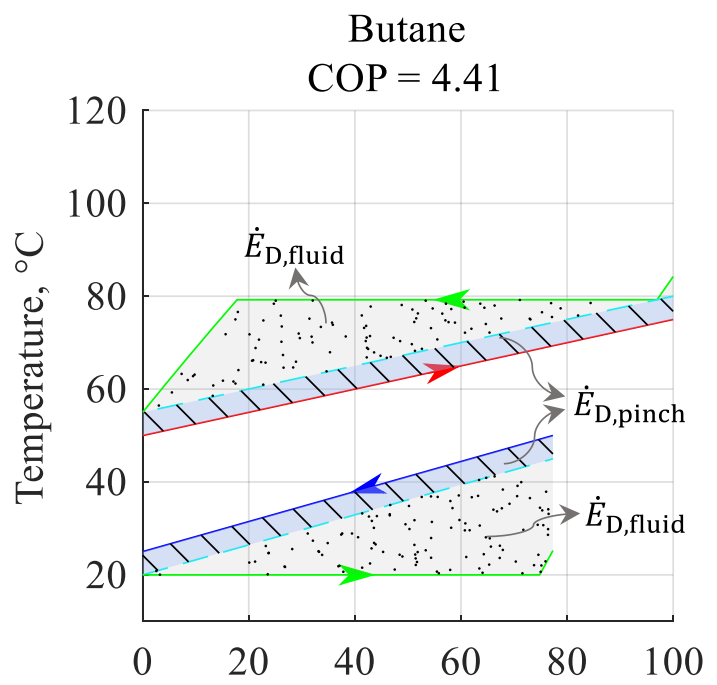

Transferred Energy per Heat Supply, \%

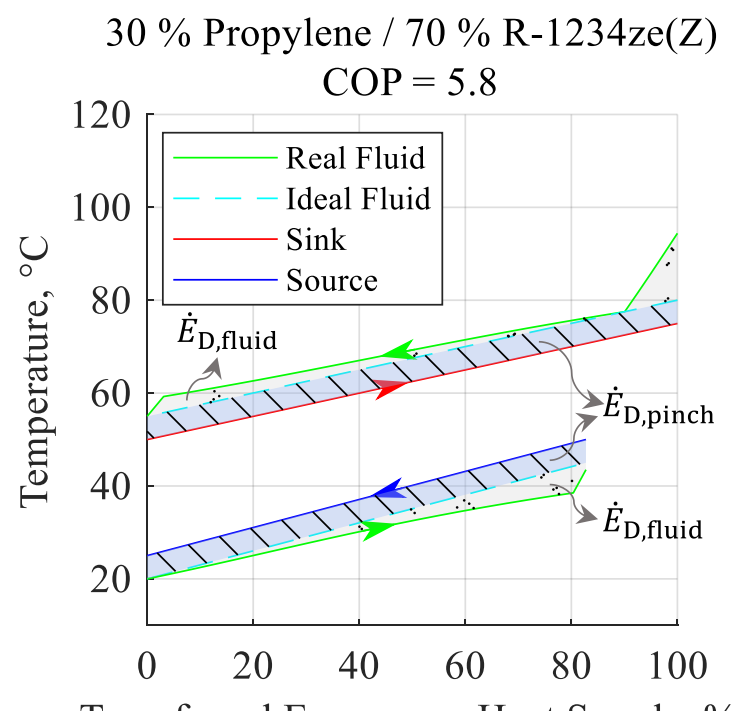

Transferred Energy per Heat Supply, \%

Figure 3. Temperature-heat-diagram of the pure fluid butane (left) and a mixture of $30 \%$ propylene $170 \%$ R-1234ze(Z) (right), including an ideal fluid as defined by the Lorenz cycle and an indication of the temperature differences associated with the fluid properties and the heat exchangers as well as the resulting exergy destruction for an exemplifying application

The exergy destruction $\dot{E}_{\mathrm{D} \text {,pinch }}$ associated with the minimum pinch point temperature difference, which results from the heat transfer of $\dot{Q}$ from a stream with a thermodynamic average temperature $\bar{T}_{\text {hot }}$ to a stream with a thermodynamic average temperature $\bar{T}_{\text {cold }}$ can be obtained from an exergy balance and is defined as shown in Eq. 3.

$$
\dot{E}_{\text {D,pinch }}=\left(1-\frac{T_{0}}{\bar{T}_{\text {hot }}}\right) \dot{Q}-\left(1-\frac{T_{0}}{\bar{T}_{\text {cold }}}\right) \dot{Q}=T_{0} \dot{Q} \frac{\bar{T}_{\text {hot }}-\bar{T}_{\text {cold }}}{\bar{T}_{\text {hot }} \bar{T}_{\text {cold }}}
$$

The exergy destruction associated with the fluid being non-ideal $\dot{E}_{\mathrm{D}, \text { fluid }}$ is determined by the difference of the total exergy destruction in the component $\dot{E}_{\mathrm{D} \text {,component }}$ and the contribution associated with the pinch point $\dot{E}_{\mathrm{D} \text {,pinch }}$.

$$
\dot{E}_{\mathrm{D}, \text { component }}=\dot{E}_{\mathrm{D}, \text { pinch }}+\dot{E}_{\mathrm{D}, \text { fluid }}
$$

The thermodynamic average temperature of a stream between temperature $T_{\mathrm{A}}$ and $T_{\mathrm{B}}$ with constant heat capacity can be calculated as $\bar{T}_{\mathrm{A}-\mathrm{B}}=\left(T_{\mathrm{A}}-T_{\mathrm{B}}\right) / \ln \left(T_{\mathrm{A}} / T_{\mathrm{B}}\right)[12]$.

The definition of the exergy destruction that was related to the heat exchanger when operating with an ideal fluid could be defined independent of medium properties. It was not possible to derive an analogous, medium-independent distinction for the compression and expansion processes. This would require an estimation of the medium properties that are ideal with respect to the compression and expansion process.

For this study, the exergy destruction in the heat exchangers transferring heat from and to the heat source and sink was distinguished as described, while the exergy destruction in the remaining components was not further distinguished.

The dead state was assumed to be at 1 bar and $1^{\circ} \mathrm{C}$. 


\subsection{Relating exergy destruction to deviations from ideal theoretical cycle in terms of COP}

Exergy based methods were found to be a meaningful tool for allocating irreversibilities to different sources. The main criterion for the evaluation of the thermodynamic performance of heat pumps remains however to be the COP. The maximum obtainable COP is defined by the Lorenz $\mathrm{COP}_{\mathrm{Lor}}$, which is obtained by the Lorenz cycle [22]. The Lorenz cycle consists of isentropic expansion and compression processes and transfers the heat at the thermodynamic average temperatures of the source and sink. The $\mathrm{COP}_{\text {Lor }}$ is accordingly solely dependent on the temperatures of the heat source and sink. The Lorenz cycle may be seen as a generalization of the Carnot cycle. For cases with no temperature glide of the sink and source the Lorenz cycle is identical to the Carnot cycle.

In order to enhance the understanding of the impact of the different rates of exergy destruction, it may be meaningful to relate these contributions to the COP. Accordingly, a relation was derived which describes the deviation of the real cycle with exergy destruction from the ideal Lorenz cycle without exergy destruction. This relation was presented in [21] and is summarized in the following. It may be noted that the supplied heat and thereby the exergy product of the heat pump was kept constant throughout this analysis. The relations were derived for an application in which all temperatures were above the dead state.

The exergy balance of the ideal Lorenz cycle without exergy destruction was defined as shown in Eq. 5. For the Lorenz cycle, the exergy product $\dot{E}_{\mathrm{P}}$ and exergy fuel $\dot{E}_{\mathrm{F}}$ are equal and defined as the sum of the power of the compressor $\dot{W}_{\text {Lor }}$ and the exergy rate supplied by the heat source $\dot{Q}_{\text {source,Lor }}\left(1-\frac{T_{0}}{\bar{T}_{\text {source }}}\right)$.

$$
\dot{E}_{\mathrm{P}}=\dot{E}_{\mathrm{F}}=\dot{E}_{\mathrm{F}, \text { comp }, \text { Lor }}+\dot{E}_{\mathrm{F}, \text { source,Lor }}=\dot{W}_{\mathrm{Lor}}+\dot{Q}_{\text {source,Lor }}\left(1-\frac{T_{0}}{\bar{T}_{\text {source }}}\right)
$$

For a real cycle, exergy destruction $\dot{E}_{\mathrm{D}}$ occurs, and the compressor power $\dot{W}_{\text {real }}$ and the exergy rate supplied by the source are adjusted by $\dot{Q}_{\text {source,real }}\left(1-\frac{T_{0}}{\bar{T}_{\text {source }}}\right)$.

$$
\dot{E}_{\mathrm{P}}+\dot{E}_{\mathrm{D}}=\dot{E}_{\mathrm{F}}=\dot{E}_{\mathrm{F}, \text { comp,real }}+\dot{E}_{\mathrm{F}, \text { source,real }}=\dot{W}_{\text {real }}+\dot{Q}_{\text {source,real }}\left(1-\frac{T_{0}}{\bar{T}_{\text {source }}}\right)
$$

As the product was maintained to be constant throughout the analysis, Eq. 6 could be derived by combining Eq. 5 and 6.

$$
\dot{W}_{\text {real }}-\dot{W}_{\text {Lor }}=\left(\dot{Q}_{\text {source,Lor }}-\dot{Q}_{\text {source,real }}\right)\left(1-\frac{T_{0}}{\bar{T}_{\text {source }}}\right)+\dot{E}_{\mathrm{D}}
$$

In addition, Eq. 8 can be derived by combining the energy balances for an ideal and a real system.

$$
\dot{Q}_{\text {source,Lor }}-\dot{Q}_{\text {source,real }}=\dot{W}_{\text {real }}-\dot{W}_{\text {Lor }}
$$

Using this relation in combination with Eq. 6 allows the definition of a relation between the additional power spent in the compressor in the real case $\left(\dot{W}_{\text {real }}-\dot{W}_{\text {Lor }}\right)$ and the exergy destruction $\dot{E}_{\mathrm{D}}$.

$$
\dot{W}_{\text {real }}-\dot{W}_{\text {Lor }}=\frac{\bar{T}_{\text {source }}}{T_{0}} \dot{E}_{\mathrm{D}}
$$


This finally enables the definition of a relation for the COP, which is solely dependent on temperatures of the heat source $\bar{T}_{\text {source }}$ and the heat sink $\bar{T}_{\text {sink }}$, the dead state temperature $T_{0}$, and the ratio of the exergy destruction and the exergy product $\frac{\dot{E}_{\mathrm{D}}}{\dot{E}_{\mathrm{P}}}$.

$$
\begin{aligned}
\mathrm{COP}=\frac{\dot{Q}_{\text {sink }}}{\dot{\mathrm{W}}_{\text {real }}} & =\frac{\dot{Q}_{\text {sink }}}{\dot{W}_{\text {Lor }}+\frac{\bar{T}_{\text {source }}}{T_{0}} \dot{E}_{\mathrm{D}}}=\frac{1}{\frac{1}{\mathrm{COP}_{\text {Lor }}}+\frac{\bar{T}_{\text {source }}}{T_{0}} \frac{\dot{E}_{\mathrm{D}}}{\dot{Q}_{\text {sink }}}} \\
& =\frac{1}{\frac{1}{\mathrm{COP}_{\text {Lor }}}+\frac{\bar{T}_{\text {source }}}{T_{0}}\left(1-\frac{T_{0}}{\bar{T}_{\text {sink }}}\right) \frac{\dot{E}_{\mathrm{D}}}{\dot{E}_{\mathrm{P}}}}
\end{aligned}
$$

For zero exergy destruction, the COP corresponds to the $\mathrm{COP}_{\mathrm{Lor}}$, while the COP decreases for an increasing exergy destruction. It may however be noted that the relation between the COP and the exergy destruction $\dot{E}_{\mathrm{D}}$ is nonlinear.

In the presented analysis, this relation was used to visualize the dependencies of the potential improvements in COP that could be obtained by reductions in exergy destruction.

\section{Results}

\subsection{Thermodynamic analysis}

This section presents a basic thermodynamic analysis of the results to outline the potentials obtainable by different mixtures of the chosen combination of propane and butane and for different cycle layouts.

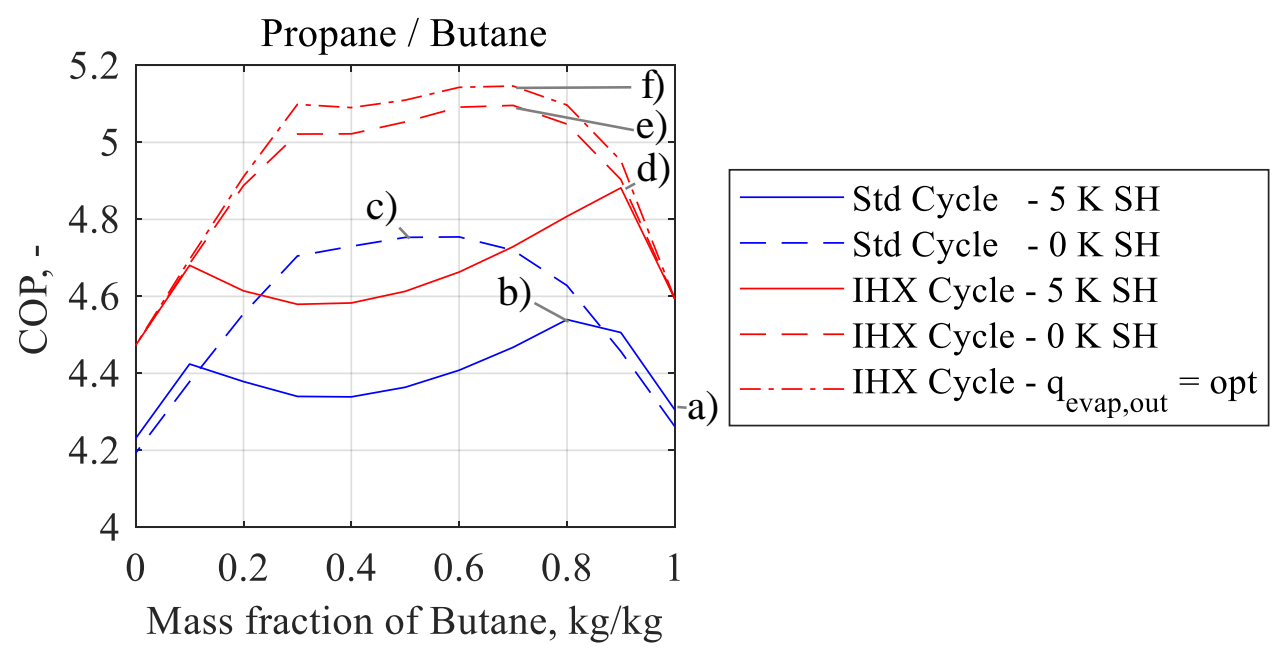

Figure 4. COP for the standard (Std) cycle and the internal heat exchanger (IHX) cycle with different fluid conditions at the outlet of the evaporator for mixtures of propane and butane

Figure 4 shows the COP for all mixtures of propane and butane depending on the mass fraction of butane for different cycle layouts. The standard cycle with a minimum required superheating of $5 \mathrm{~K}$ inside the evaporator was included as a baseline. It was furthermore assumed, that the required superheating inside the evaporator could be reduced by e.g., an advanced control of the evaporator outlet enabled by the temperature glide of the mixtures, without adding a component. In addition to the standard cycle, the IHX-cycle was presented for three different assumptions. It was assumed that the outlet of the evaporator is i) superheated by $5 \mathrm{~K}$, ii) in saturated conditions and iii) at an optimized outlet quality. Based on these results, six representative cases were selected for further evaluation and summarized in Table 1 and visualized by means of temperature heat diagrams in Figure 5. 


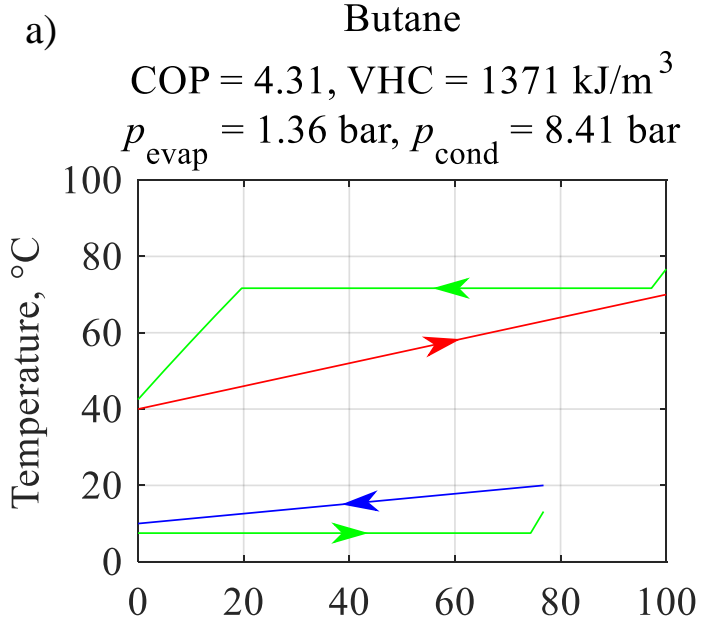

Transferred Energy per Heat Supply, \%
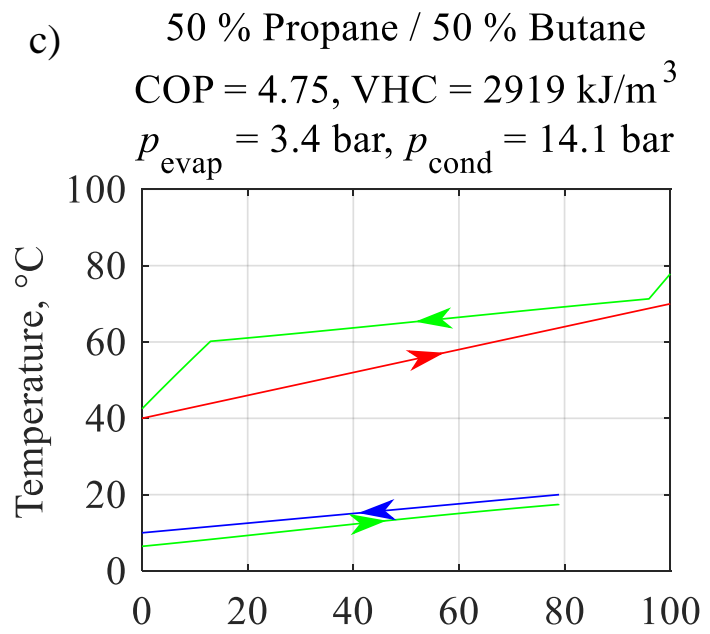

Transferred Energy per Heat Supply, \%

e) $\quad 30 \%$ Propane $/ 70 \%$ Butane

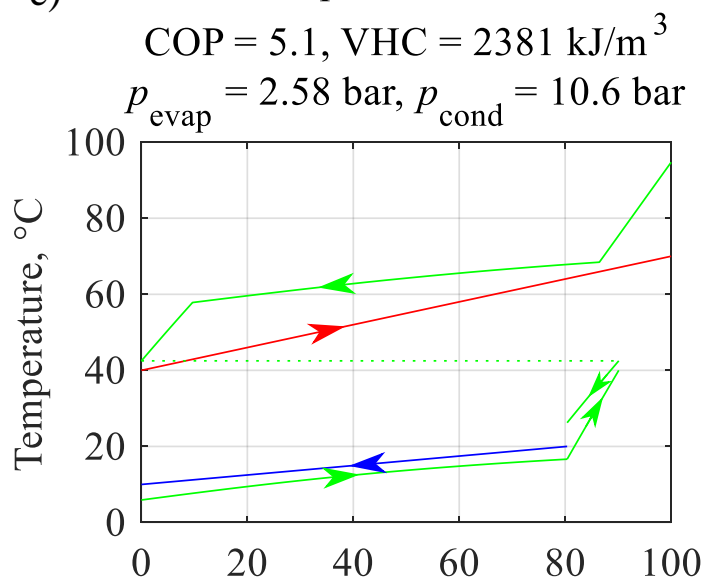

Transferred Energy per Heat Supply, \%

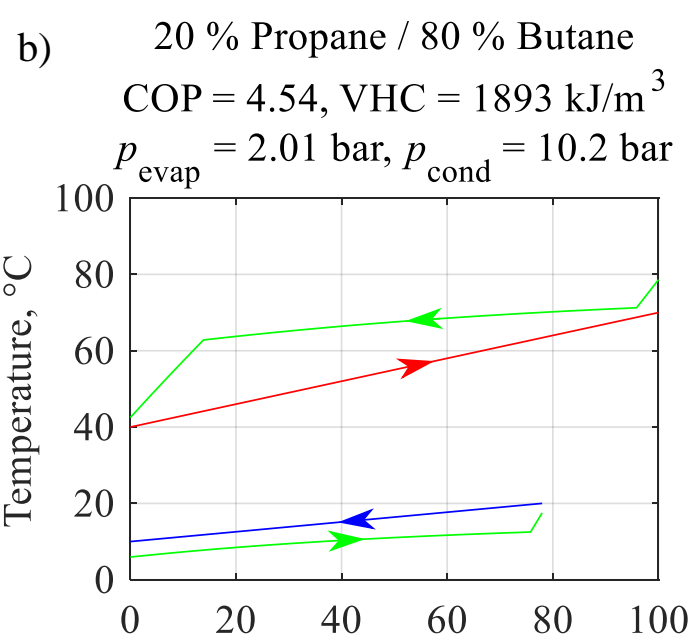

Transferred Energy per Heat Supply, \%

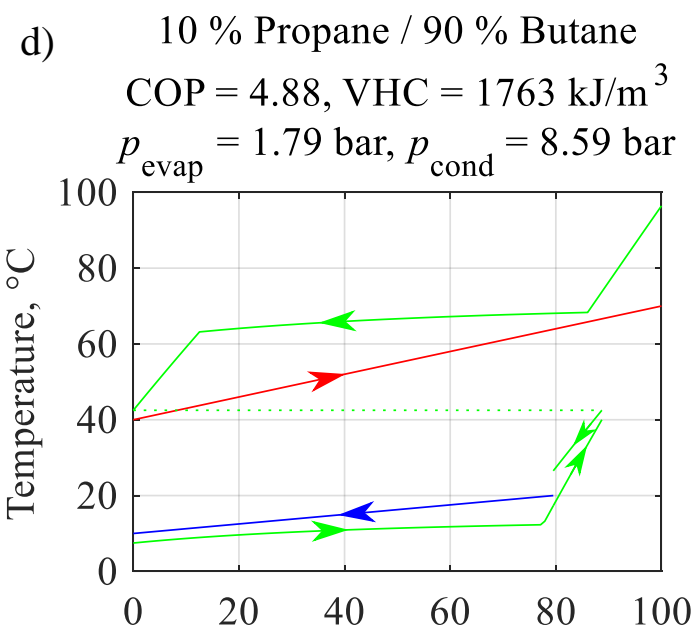

Transferred Energy per Heat Supply, \%

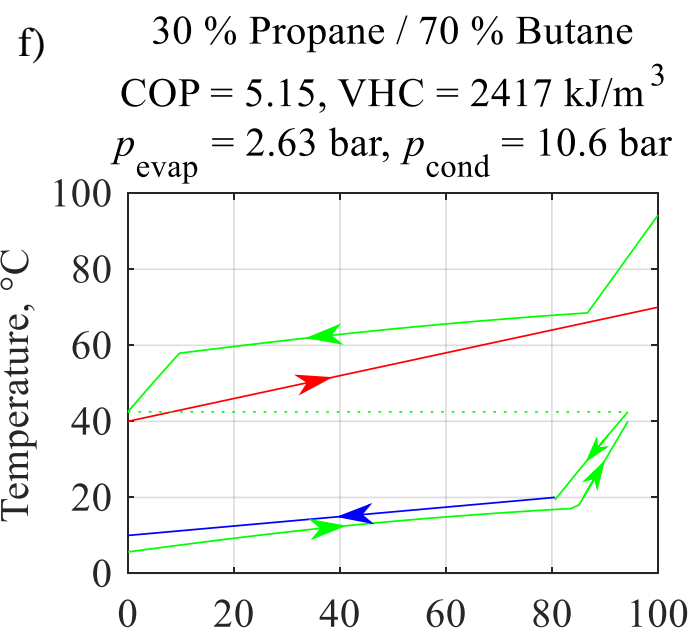

Transferred Energy per Heat Supply, \%

Figure 5. Temperature-heat-diagrams for cases a) to f) from Table 1 
Table 1. Summary of COP, volumetric heating capacity VHC and pressure levels for selected cases

\begin{tabular}{cccccccc}
\hline Case & Cycle & $\begin{array}{c}\text { Condition at } \\
\text { evaporator outlet }\end{array}$ & Medium (Example) & $\begin{array}{c}\text { COP, } \\
-\end{array}$ & $\begin{array}{c}\mathrm{VHC}, \\
\mathrm{kJ} / \mathrm{m}^{3}\end{array}$ & $\begin{array}{c}p_{\text {evap, }}, \\
\text { bar }\end{array}$ & $\begin{array}{c}p_{\text {cond }}, \\
\text { bar }\end{array}$ \\
\hline a) & Std-cycle & Superheated by 5 K & 20\% Propane / 80 \% Butane & 4.51 & 1371 & 1.4 & 8.4 \\
b) & & & Butane & 1893 & 2.0 & 10.2 \\
c) & Std-cycle & Superheated by 0 K & $50 \%$ Propane / 50 \% Butane & 4.75 & 2919 & 3.4 & 14.1 \\
d) & IHX-cycle & Superheated by 5 K & 10\% Propane / 90 \% Butane & 4.88 & 1763 & 1.8 & 8.6 \\
e) & IHX-cycle & Superheated by 0 K & 30\% Propane / 70 \% Butane & 5.10 & 2381 & 2.6 & 10.6 \\
f) & IHX-cycle & Optimized quality & 30\% Propane / 70 \% Butane & 5.15 & 2417 & 2.6 & 10.6 \\
\hline
\end{tabular}

The diagram shows a COP of 4.3 for butane at (a), which is slightly higher than for propane for the standard cycle with $5 \mathrm{~K}$ superheating inside the evaporator. For the same cycle configuration, the COP could be increased to 4.5 for $20 \%$ propane / $80 \%$ butane (b). Assuming that the superheating inside the evaporator could be reduced benefitted mainly the mixtures with a good glide match on the source side and yielded a COP of 4.8 for $50 \%$ propane / $50 \%$ butane.

The results for the configuration in which an internal heat exchanger was considered and a minimum required superheating of $5 \mathrm{~K}$ inside the evaporator was assumed were similar to the standard cycle with minimum $5 \mathrm{~K}$ superheating inside the evaporator with a relatively constant offset of 0.25 in COP. This enabled a COP of 4.9 for $10 \%$ propane / $90 \%$ butane.

The reduction of the required superheating inside the evaporator showed a similar increase in COP for the IHX-cycle as it did for the standard cycle. The mixture $30 \%$ propane $/ 70 \%$ butane reached a COP of 5.1 with saturated conditions at the outlet of the evaporator, while it reached 5.15 for an optimized outlet quality. It may furthermore be noted that a higher concentration of propane yielded higher pressure levels and higher volumetric heating capacities.

Figure 5 shows the temperature-heat-diagrams for the cases described in Table 1. In case a), butane showed a mismatch of the temperature profiles on both the heat source and sink side. The temperature match on the source side was improved for all other cases, while an optimal match is obtained for the cases in which the superheating inside the evaporator was omitted. On the sink side, the temperature match was improved compared to butane, while a certain mismatch remained.

\subsection{Exergy Analysis}

The thermodynamic analysis documented considerable performance improvement potentials associated with the use of zeotropic working fluid mixtures for the selected case. This section presents the results of an exergy analysis and relates the contributions of exergy destruction to potential increases in COP. The exergy destruction in the heat exchangers was furthermore distinguished into a contribution associated with the pinch point temperature difference that could not be avoided with an ideal fluid and another contribution accounting for the fluid being non-ideal.

Figure 6 shows the different sources of exergy destruction $\dot{E}_{\mathrm{D}}$ in relation to the exergy product $\dot{E}_{\mathrm{P}}$ and relates these to the COP for the selected six cases. The COP approaches the Lorenz COP $\mathrm{Lor}_{\text {for }}$ zero exergy destruction and decreases non-linearly for an increasing exergy destruction. The potential increase in COP associated with the reduction of a unit of exergy destruction is accordingly higher for lower values of absolute total exergy destruction.

The exergy destruction associated with the pinch point temperature differences remains constant for the condenser and increases slightly for the evaporator. The slight increase results from the increasing heat flow rate in the evaporator at higher COPs. The exergy destruction in relation to the exergy product occurring in the compressor decreases from $22 \%$ in a) to $18 \%$ in e), which might result from decreasing temperature differences between compressor inlet and outlet. The irreversibilities associated with the throttling valve decrease considerably due to the introduction of the internal heat exchanger, which compensates the additional exergy destruction resulting from the heat transfer. 
$\boxminus$ Condenser (Pinch)

ه Throttling Valve

$\square$ Evaporator (Fluid)

$$
\begin{array}{ll}
\Xi \text { Evaporator (Pinch) } & \varangle \text { Compressor } \\
\checkmark \text { Internal HX } & \square \text { Condenser (Fluid) }
\end{array}
$$

$\begin{array}{llllllllll}0 & 10 & 20 & 30 & 40 & 50 & 60 & 70 & 80\end{array}$

a) Std-Cycle, $5 \mathrm{~K} \mathrm{SH}$ Butane

b) Std-Cycle, $5 \mathrm{~K} \mathrm{SH}$

$20 \%$ Propane / $80 \%$ Butane

c) Std-Cycle, $0 \mathrm{~K} \mathrm{SH}$

$50 \%$ Propane / $50 \%$ Butane

d) IHX-Cycle, $5 \mathrm{~K} \mathrm{SH}$

$10 \%$ Propane / $90 \%$ Butane

e) IHX-Cycle, $0 \mathrm{~K} \mathrm{SH}$

$30 \%$ Propane $/ 70 \%$ Butane

f) IHX-Cycle, $q_{\text {evap,out }}=$ opt

$30 \%$ Propane $/ 70 \%$ Butane
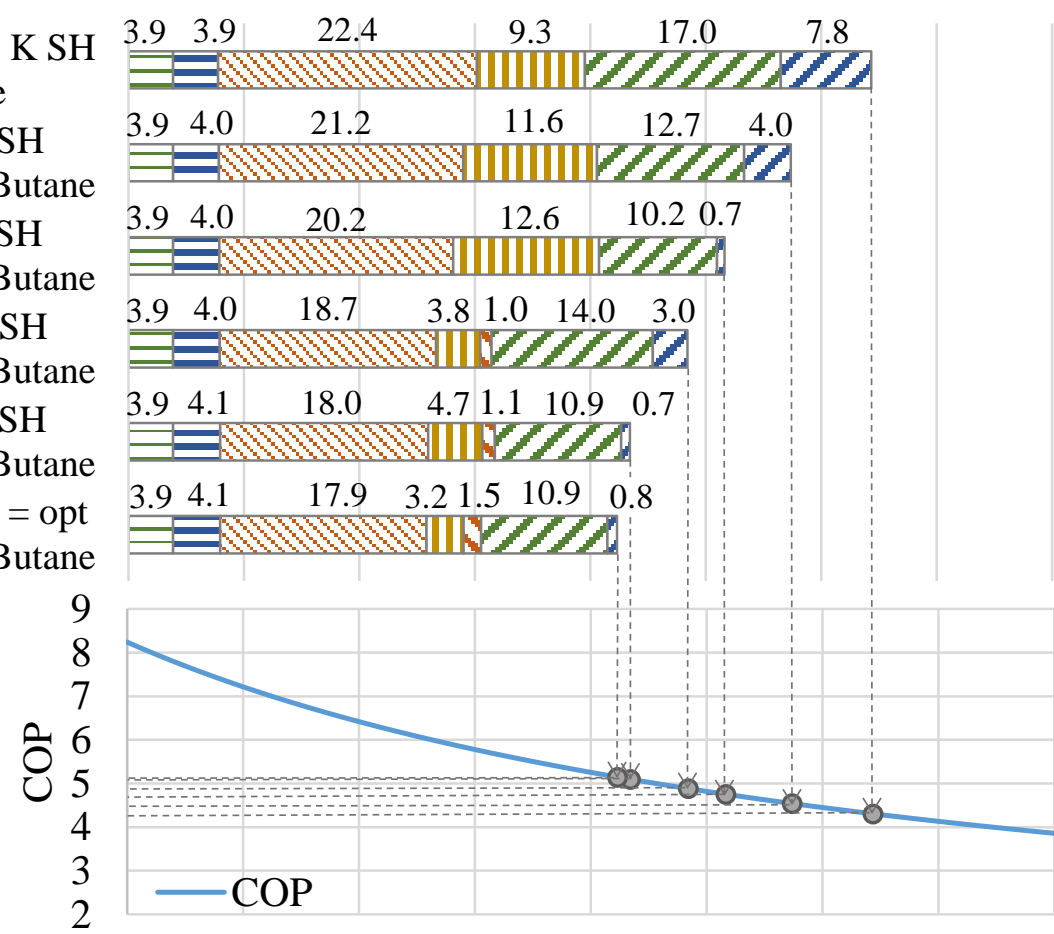

Figure 6. Overview of the exergy destruction per exergy product in relation to the COP for all considered cases

The exergy destruction associated with the temperature profile of a real fluid deviating from an ideally matched working fluid decreases from $17 \%$ for a) to $11 \%$ for e), in the condenser, while it could be reduced to below $1 \%$ for the evaporator. These findings are furthermore consistent with the observations from the temperature-heat-diagrams.

\section{Discussion}

The study presented an exergy-based analysis of different working fluids and different cycle layouts. It highlighted the different contributions of irreversibilities in terms of exergy destruction and related these contributions to the deviations in COP from the theoretically ideal Lorenz COP $\mathrm{Lor}_{\text {. }}$ The study was based on a specific example, and it was limited to the analysis of binary mixtures of propane and butane. It may be noted that the mixture of propane/butane was chosen as an example for the demonstration of the method. The temperature glides of these mixtures are relatively similar in both evaporation and condensation. This yields a moderate mismatch in the sink side, which cannot be avoided for the described case study considering propane/butane. Zühlsdorf et al. [6] outlined that the improved temperature glide matches might be obtained by other fluids, which are e.g., operating with a condenser pressure close to or above the critical pressure.

The exergy destruction in the heat source and heat sink heat exchangers was distinguished into two contributions. One contribution was associated with the heat exchanger equipment and the other with the fluid being nonideal. The exergy destruction associated to the heat exchanger equipment was considered to be unavoidable by optimizing the working fluid and the cycle layout. The results indicated that these contributions remained constant and were only impacted by the different heat flow rates in the evaporator resulting from the variations in COP and the constant heat sink capacity during the study. This distinction was found to be a meaningful quantification of the exergy 
destruction, which could be reduced by temperature glide matching with a sophisticated working fluid choice.

The ideal fluid with respect to the heat transfer process was identified as a fluid with an optimally matched temperature profile. This enabled determining the exergy destruction associated with the component when operating with an ideal fluid, without requiring specific medium properties. For the case of the compression and expansion process, it was not possible to analogously derive an ideal case, which was independent of medium properties. An estimation of the exergy destruction related to the compression and expansion process working with an ideal fluid might however be obtained by introducing limitations for the medium properties that determine the fluid performance. Such limitations may be derived from theoretical observations or the variety of existing fluids.

\section{Conclusions}

The article presented an exergy-based analysis of different working fluids and cycle layouts and related the exergy destruction to deviations from a theoretically ideal Lorenz cycle in terms of COP. The study was based on a case study with fixed inlet and outlet conditions considering different mixtures of propane/butane.

The exergy destruction in the heat exchangers of heat source and sink were, in addition to a conventional exergy analysis, distinguished into a contribution solely resulting from the pinch point temperature difference for an ideally matched working fluid and a contribution accounting for the fluid being non-ideal. This distinction was found suitable for quantifying the optimization potential associated with temperature glide matching using zeotropic working fluids.

Furthermore, the exergy destruction was related to deviations from the Lorenz cycle in terms of COP. This enabled a more intuitive interpretation of the exergy destruction and the corresponding impact on the thermodynamic performance.

\section{Acknowledgments}

This research project is financially funded by The Danish Council for Strategic Research in Sustainable Energy and Environment, under the project title: "THERMCYC - Advanced thermodynamic cycles utilizing low-temperature heat sources".

\section{Nomenclature}

Latin symbols:

\begin{tabular}{|c|c|c|c|}
\hline $\begin{array}{l}\mathrm{COP} \\
\dot{E}\end{array}$ & $\begin{array}{l}\text { Coefficient of performance, - } \\
\text { Exergy flow rate, } \mathrm{kW}\end{array}$ & $\bar{T}$ & $\begin{array}{l}\text { Thermodynamic average } \\
\text { temperature, }{ }^{\circ} \mathrm{C}\end{array}$ \\
\hline$p$ & Pressure, bar & $\dot{V}$ & Volume flow rate, $\mathrm{m}^{3} / \mathrm{s}$ \\
\hline$\dot{Q}$ & Heat flow rate, $\mathrm{kW}$ & VHC & $\begin{array}{l}\text { Volumetric heating capacity, } \\
\mathrm{kJ} / \mathrm{m}^{3}\end{array}$ \\
\hline$T$ & Temperature, ${ }^{\circ} \mathrm{C}$ & $\dot{W}$ & Power, kW \\
\hline
\end{tabular}

Subscripts and superscripts:

$\begin{array}{llll}\text { Comp } & \text { Compressor } & \text { Lor } & \text { Lorenz cycle } \\ \text { cold } & \text { Cold side } & \text { P } & \text { Product } \\ \text { cond } & \text { Condensation } & \text { pinch } & \text { Pinch } \\ \text { D } & \text { Destruction } & \text { real } & \text { Real cycle } \\ \text { evap } & \text { Evaporation } & \text { Sink } & \text { Sink } \\ \text { F } & \text { Fuel } & \text { Source } & \text { Source } \\ \text { fluid } & \text { Fluid } & 0 & \text { Dead state } \\ \text { hot } & \text { Hot side } & 1 \ldots 8 & \text { State points }\end{array}$




\section{References}

[1] Domanski PA, Steven Brown J, Heo J, Wojtusiak J, McLinden MO. A thermodynamic analysis of refrigerants: Performance limits of the vapor compression cycle. International Journal of Refrigeration 2014;38:71-9. doi:10.1016/j.ijrefrig.2013.09.036.

[2] McLinden MO, Kazakov AF, Steven Brown J, Domanski PA. A thermodynamic analysis of refrigerants: Possibilities and tradeoffs for Low-GWP refrigerants. International Journal of Refrigeration 2014;38:80-92. doi:10.1016/j.ijrefrig.2013.09.032.

[3] McLinden MO, Brown JS, Brignoli R, Kazakov AF, Domanski PA. Limited options for lowglobal-warming-potential refrigerants. Nature Communications 2017;8:1-9. doi:10.1038/ncomms14476.

[4] Harby K. Hydrocarbons and their mixtures as alternatives to environmental unfriendly halogenated refrigerants: An updated overview. Renewable and Sustainable Energy Reviews 2017;73:1247-64. doi:10.1016/j.rser.2017.02.039.

[5] Zühlsdorf B, Jensen JK, Elmegaard B. Heat pump working fluid selection - Economic and thermodynamic comparison of criteria and boundary conditions. International Journal of Refrigeration 2019;98:500-13. doi:10.1016/j.jirefrig.2018.11.034.

[6] Zühlsdorf B, Jensen JK, Cignitti S, Madsen C, Elmegaard B. Analysis of temperature glide matching of heat pumps with zeotropic working fluid mixtures for different temperature glides. Energy 2018;153:650-60. doi:https://doi.org/10.1016/j.energy.2018.04.048.

[7] Jensen JK, Markussen WB, Reinholdt L, Elmegaard B. On the development of high temperature ammonia-water hybrid absorption-compression heat pumps. International Journal of Refrigeration 2015;58:79-89. doi:10.1016/j.ijrefrig.2015.06.006.

[8] Van De Bor DM, Infante Ferreira CA, Kiss AA. Optimal performance of compressionresorption heat pump systems. Applied Thermal Engineering 2014;65:219-25. doi:10.1016/j.applthermaleng.2013.12.067.

[9] Gudjonsdottir V, Infante Ferreira CA, Rexwinkel G, Kiss AA. Enhanced performance of wet compression-resorption heat pumps by using $\mathrm{NH} 3-\mathrm{CO} 2-\mathrm{H} 2 \mathrm{O}$ as working fluid. Energy 2017;124:531-42. doi:10.1016/j.energy.2017.02.051.

[10] Morosuk T, Tsatsaronis G, Schult M. Conventional and advanced exergetic analyses: Theory and application. Arabian Journal for Science and Engineering 2013;38:395-404. doi:10.1007/s 13369-012-0441-9.

[11] Tsatsaronis G, Morosuk T. Advanced thermodynamic (exergetic) analysis. Journal of Physics: Conference Series 2012;395. doi:10.1088/1742-6596/395/1/012160.

[12] Bejan A, Tsatsaronis G, Moran M. Thermal Design and Optimization. 1996. doi:10.1016/S0140-7007(97)87632-3.

[13] Tsatsaronis G, Park M. On avoidable and unavoidable exergy destructions and investment costs in thermal systems Tsatsaronis, G. and Park, M.-H. Energy Conversion and Management, 2002, 43, (9-12), 1259-1270. Fuel and Energy Abstracts 2003;44:102. doi:10.1016/S0140-6701(03)90782-X.

[14] Kelly S, Tsatsaronis G, Morosuk T. Advanced exergetic analysis: Approaches for splitting the exergy destruction into endogenous and exogenous parts. Energy 2009;34:384-91. doi:10.1016/j.energy.2008.12.007.

[15] Morosuk T, Tsatsaronis G. A new approach to the exergy analysis of absorption refrigeration machines. Energy 2008;33:890-907. doi:10.1016/j.energy.2007.09.012.

[16] Jensen JK, Markussen WB, Reinholdt L, Elmegaard B. Exergoeconomic optimization of an ammonia-water hybrid absorption-compression heat pump for heat supply in a spray-drying facility. International Journal of Energy and Environmental Engineering 2015;6:195-211. doi:10.1007/s40095-015-0166-0. 
[17] Morosuk T, Tsatsaronis G. Advanced exergetic evaluation of refrigeration machines using different working fluids. Energy 2009;34:2248-58. doi:10.1016/j.energy.2009.01.006.

[18] Zühlsdorf B, Meesenburg W, Ommen TS, Thorsen JE, Markussen WB, Elmegaard B. Improving the performance of booster heat pumps using zeotropic mixtures. Energy 2018;154:390-402. doi:https://doi.org/10.1016/j.energy.2018.04.137.

[19] Lemmon EW, Huber ML, McLinden MO. NIST Standard Reference Database 23: Reference Fluid Thermodynamic and Transport Properties-REFPROP, Version 9.12013.

[20] Zühlsdorf B, Jensen JK, Elmegaard B. Numerical models for the design and analysis of heat pumps with zeotropic mixtures, Rev01 2018. doi:https://doi.org/10.11583/DTU.6825443.

[21] Zühlsdorf B. High-performance heat pump systems - Enhancing performance and range of heat pump systems for industry and district heating. Technical University of Denmark, 2019.

[22] Lorenz H. Beiträge zur Beurteilung von Kühlmaschinen. Zeitschrift Des VDI 1894;38:62-8. 